\title{
Staffordshire Bull Terrier
}

National Cancer Institute

\section{Source}

National Cancer Institute. Staffordshire Bull Terrier. NCI Thesaurus. Code C53733.

The Staffordshire Bull Terrier is a powerful and muscular dog, very strong for his size with a broad head and very strong jaws. The ears are either rose or half-pricked. The short coat is soft and close and comes in black, blue, fawn or brindle, often with white markings. Height: 13-16 inches (33-41 cm.) Weight: 23-38 pounds (11-17 kg.) 